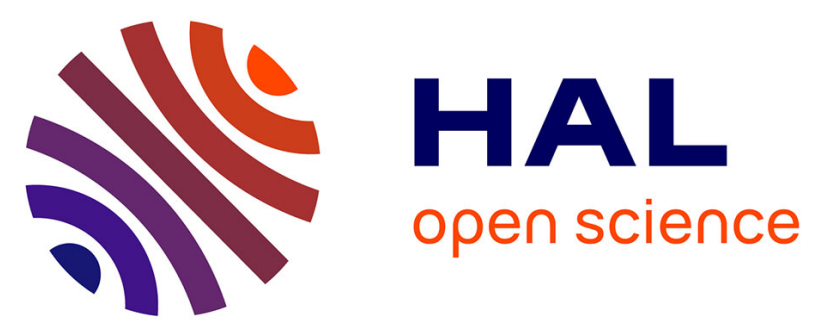

\title{
Étude de films bleu de Prusse/Nafion ® 117 par microbalance à quartz en régime dynamique
}

Loan To Thi Kim, Claude Gabrielli, Hubert Perrot, F. Vicente, J.

Garcia-Jareno, D. Giménez-Romero

\section{- To cite this version:}

Loan To Thi Kim, Claude Gabrielli, Hubert Perrot, F. Vicente, J. Garcia-Jareno, et al.. Étude de films bleu de Prusse/Nafion ( 117 par microbalance à quartz en régime dynamique. Matériaux \& Techniques, 2007, XXe Forum sur les impédances électrochimiques / 20th Electrochemical Impedance Forum, 95, pp.435-442. 10.1051/mattech:2008040 . hal-01731400

\section{HAL Id: hal-01731400 https: / hal.sorbonne-universite.fr/hal-01731400}

Submitted on 14 Mar 2018

HAL is a multi-disciplinary open access archive for the deposit and dissemination of scientific research documents, whether they are published or not. The documents may come from teaching and research institutions in France or abroad, or from public or private research centers.
L'archive ouverte pluridisciplinaire HAL, est destinée au dépôt et à la diffusion de documents scientifiques de niveau recherche, publiés ou non, émanant des établissements d'enseignement et de recherche français ou étrangers, des laboratoires publics ou privés. 


\title{
Étude de films bleu de Prusse/Nafion ${ }^{\circledR}$ 117 par microbalance à quartz en régime dynamique
}

\author{
L. To Thi Kim ${ }^{1}$, C. Gabrielli ${ }^{1}$, H. Perrot ${ }^{1}$, F. Vicente ${ }^{2}$, J.J. García-Jareño ${ }^{2}$ \\ et $D$. Giménez-Romero ${ }^{2}$ \\ 1 Université P. et M. Curie, UPR 15 du CNRS-LISE, 4 place Jussieu, 75252 Paris, France \\ e-mail : tloan20231982@yahoo.com ; claude.gabrielli@upmc.fr; hubert.perrot@upmc.fr \\ 2 Departament Química Física. /Dr. Moliner, 50, 46100 Burjassot, València, Espagne \\ e-mail : Francisco.Vicente@uv.es; Jose.J.Garcia@uv.es; giroda@uv.es
}

Mots-clés :

Nafion; microbalance; electrogravimétrie; bleu de Prusse

\section{Key words:}

Nafion; microbalance; electrogravimetry; prussian blue
Résumé - La technique d'électrogravimétrie, basée sur une microbalance à quartz en régime dynamique, permet d'accéder à des informations cinétiques réactionnelles de films électroactifs. Le mouvement des espèces (chargées ou non chargées), qui entrent ou sortent du film quand il est réduit ou oxydé, est analysé. L'utilisation d'un médiateur comme le bleu de Prusse (PB) entre l'électrode de travail de la microbalance à quartz et un film de Nafion ${ }^{\circledR}$ permet de réaliser l'étude de ce dernier. Les réponses électrochimiques de la structure PB/ Nafion ${ }^{\circledR}$ sont obtenues dans deux milieux différents $\mathrm{HCl} \mathrm{0,05M}$ et $\mathrm{KCl} 0,05 \mathrm{M}+\mathrm{HCl} 0,05 \mathrm{M}$. Ainsi, malgré le caractère conducteur protonique du Nafion ${ }^{\circledR}$, la technique d'électrogravimétrie a révélé que d'autres espèces, anion, solvant libre et autres cations, pouvaient interagir avec ce film.

Abstract - Study of Prussian blue/Nafion ${ }^{\circledR} 117$ films by fast quartz crystal microbalance. Ac-electrogravimetry technique, based on a quartz crystal microbalance, allows kinetic subleties for electroactive films to be obtained. The motion of charged or non charged species, inserted or expelled during the redox switching of the film, is directly analysed. A mediator film is used between the working electrode of the microbalance and the Nafion ${ }^{\circledR}$ layer in order to perform the electrochemical measurements. The electrochemical and gravimetric response were obtained in two different medium: $\mathrm{HCl} 0.05 \mathrm{M}$ and $\mathrm{KCl} 0.05 \mathrm{M}$ $+\mathrm{HCl} 0.05 \mathrm{M}$. Thus, even with well known protonic behaviour of the Nafion ${ }^{\circledR}$ films, this technique of fast microbalance pointed out the contribution of different species as anions, free solvent and also different cation.

tant un polymère de l'acide poly(perfluorosulfonique), le Nafion ${ }^{\circledR}$ se présente depuis 1960 [1] comme une excellente membrane échangeuse de protons, pour des applications dans le domaine des piles à combustibles de type PEMFC (en anglais PEMFC : Proton Echange Membrane Fuel Cell), grâce à sa grande stabilité thermique et mécanique. À l'heure actuelle, il y a eu beaucoup d'études menées sur le Nafion ${ }^{\circledR}$ afin de mieux connaître la relation entre ses propriétés physicochimiques et sa structure ainsi que sur les phénomènes de transfert de matière entre celui-ci et un électrolyte. Malheureusement, en ce qui concerne ce dernier point, il reste encore du travail à faire, notamment lorsque le film est au contact avec des électrolytes complexes. Au laboratoire, la technique d'électrogravimétrie a été envisagée afin de mettre en évidence ces mouvements d'espèces (chargées comme les cations, les anions et non chargées comme le solvant libre) à l'interface film/électrolyte et de les identifier.

Dans nos précédents travaux, la technique d'électrogravimétrie a été mise au point pour étudier des films électroactifs, comme le polypyrrole $[2,3]$ ou le bleu de Prusse [4-6]. En mesurant simultanément l'impédance électrochimique (concernant l'aspect charge électrique) et la fonction de transfert électrogravimétrique (concernant l'aspect variation de masse), le rôle de chaque ion participant à la compensation de charge, à chaque potentiel imposé, a été établi lorsque le film est électroactif. 
En tant que conducteur purement ionique, le Nafion ${ }^{\circledR}$ ne peut être étudié directement sur l'électrode de travail en or de la microbalance à quartz, un médiateur entre celle-ci et le Nafion ${ }^{\circledR}$ est nécessaire. Dans le cadre de notre travail, un film de bleu de Prusse (PB) a donc été choisi comme film électroactif intermédiaire. Par électrogravimétrie, des études de films $\mathrm{PB}\left(\mathrm{Fe}^{\mathrm{III}} \mathrm{Fe}^{\mathrm{II}}(\mathrm{CN})_{6}\right)$ ont été réalisées en milieu $\mathrm{KCl}$ à des $\mathrm{pH}$ acides [4-6]. Les résultats obtenus montrent que pour des potentiels cathodiques, $\mathrm{K}^{+}$ intervient majoritairement tandis que pour des potentiels anodiques, la contribution d'hydronium, $\mathrm{H}_{3} \mathrm{O}^{+}$, devient importante en considérant le transfert du cation comme un processus prédominant durant l'oxydation du film.

Ce travail comprend, dans premier temps, une approche théorique ainsi que le calcul/simulation des différentes fonctions de transfert obtenues. Puis, cette approche sera utilisée pour comprendre les mécanismes de transfert des espèces entre la structure $\mathrm{PB} / \mathrm{Nafion}{ }^{\circledR}$ et l'électrolyte.

\section{Théorie}

Dans cette approche, le transfert de charge à l'interface électrode/film ainsi que le transport de matière dans le film et dans la solution sont considérés comme des étapes non limitantes au cours du processus réactionnel. Cette hypothèse peut aisément se justifier pour des films fins.

\section{Transfert des espèces}

La figure 1 présente un schéma des différentes interfaces électrode/film/solution. Un milieu classique sera considéré avec des cations $\left(c_{i}\right)$, des anions $\left(a_{k}\right)$ et un solvant $(s)$ qui peut éventuellement accompagner les espèces chargées ou se déplacer seul.

Par définition, le flux de l'espèce $\alpha\left(c_{i}\right.$, $a_{k}$ ou $\left.s\right), J_{\alpha}$, sera considéré comme positif si l'espèce est expulsée du film :

$$
J_{\alpha}>0 \quad \text { pour } \quad x>0 .
$$

Le mécanisme de dopage d'un film électroactif peut être décrit par les réactions suivantes :

$\langle P\rangle+e^{-}+C^{+} \underset{k_{c}^{\prime}}{\stackrel{k_{c}}{\longrightarrow}}\left\langle P, C^{+}\right\rangle$et $\langle P\rangle+A^{-} \underset{k_{a}}{\stackrel{k_{a}^{\prime}}{\longrightarrow}}\langle P, A\rangle+e^{-}$

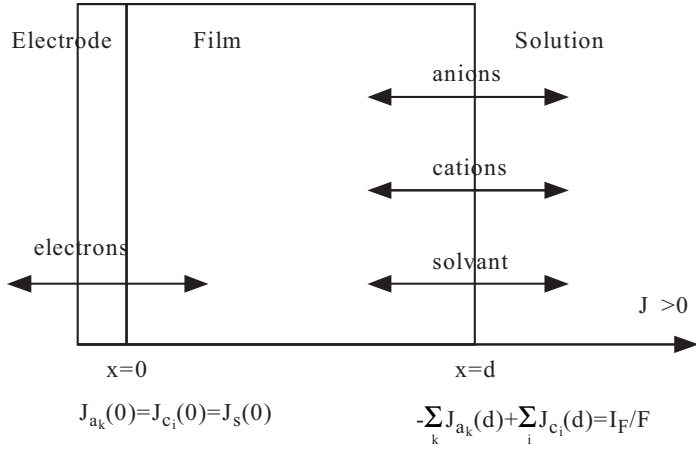

Fig. 1. Représentation schématique des interfaces électrode/film et film/solution.

Fig. 1. Schematic representation of the electrode/film and film/solution interfaces.

où $\langle P\rangle$ est le site électroactif du film, $\left\langle P, C^{+}\right\rangle$, et $\left\langle P, A^{-}\right\rangle$les cations et anions insérés dans le film.

En utilisant les lois de la cinétique hétérogène, le flux molaire instantané de l'espèce $\alpha$ est supposé tel que :

$$
\begin{aligned}
J_{\alpha}(d) & =-d \frac{d C_{\alpha}}{d t} \\
& =k_{\alpha}\left(C_{\alpha}-C_{\alpha_{\min }}\right)-k_{\alpha}^{\prime}\left(C_{\alpha_{\max }}-C_{\alpha}\right) C_{\alpha_{s o l}}
\end{aligned}
$$

où $\frac{d C_{\alpha}}{d t}>0$ pour les espèces insérées, $d$ est l'épaisseur du film, $C_{\alpha_{\max }}$ est la concentration maximale de sites dans le film pour l'insertion d'espèces, $C_{\alpha_{\min }}$ est la concentration minimale de sites occupés dans le film, $C_{\alpha_{s o l}}$ est la concentration de l'espèce, $\alpha$, dans la solution et $k_{\alpha}$ et $k_{\alpha}^{\prime}$ sont les constantes de vitesse qui suivent une loi de Tafel.

\section{Régime dynamique}

Sous l'effet d'une perturbation sinusoïdale en potentiel de faible niveau, $\Delta E$, imposée à l'ensemble des interfaces métal/film/électrolyte, des fluctuations sinusoïdales de concentration, $\Delta C_{\alpha}$, et de flux, $\Delta J_{\alpha}$, sont observées. Cela peut s'écrire de la façon suivante :

$$
\begin{aligned}
& \Delta J_{\alpha}=-j \omega d \Delta C_{\alpha}=K_{\alpha} \Delta C_{\alpha}(d)+G_{\alpha} \Delta E \\
& \alpha=c_{i}, a_{i}, s
\end{aligned}
$$

où

$$
G_{\alpha}=b_{\alpha} k_{\alpha}\left(C_{\alpha}-C_{\alpha_{\min }}\right)-b_{\alpha}^{\prime} k_{\alpha}^{\prime}\left(C_{\alpha_{\max }}-C_{\alpha}\right) C_{\alpha_{s o l}}
$$

$$
K_{\alpha}=k_{\alpha}^{\prime}+k_{\alpha}^{\prime} C_{\alpha_{s o l}}
$$


et $G_{\alpha}<0$ pour insérer des espèces et $G_{\alpha}>0$ pour l'expulsion.

D'après l'équation (3) il vient :

$$
\frac{\Delta C_{\alpha}}{\Delta E}(\omega)=\frac{-G_{\alpha}}{j \omega d+K_{\alpha}} .
$$

À partir de cette équation $\frac{\Delta C_{\alpha}}{\Delta E}(\omega)$, les quatre fonctions de transfert ont été calculées théoriquement:

- l'impédance électrochimique, $\frac{\Delta E}{\Delta I}(\omega)$, qui tient compte de la capacité de double couche $C_{d l}$ vaut :

$$
\begin{aligned}
& \frac{\Delta E}{\Delta I}(\omega)= \\
& \frac{1}{j \omega C_{d l}+j \omega d\left[\sum_{i} z_{c_{i}} F \frac{G_{c_{i}}}{j \omega d+K_{c_{i}}}-\sum_{k} z_{a_{k}} F \frac{G_{a_{k}}}{j \omega d+K_{a_{k}}}\right]}
\end{aligned}
$$

- la fonction de transfert électrogravimétrique, $\frac{\Delta m}{\Delta E}(\omega)$ :

$$
\begin{aligned}
\frac{\Delta m}{\Delta E}(\omega)= & d\left(\sum_{i} m_{c_{i}} \frac{-G_{c_{i}}}{j \omega d+K_{c_{i}}}\right. \\
& \left.+\sum_{k} m_{a_{k}} \frac{-G_{a_{k}}}{j \omega d+K_{a_{k}}}+m_{s} \frac{-G_{s}}{j \omega d+K_{s}}\right)
\end{aligned}
$$

- la fonction de transfert charge/potentiel, $\frac{\Delta q}{\Delta E}(\omega)$ :

$$
\begin{aligned}
& \frac{\Delta q}{\Delta E}(\omega)=\frac{1}{j \omega} \frac{\Delta I_{F}}{\Delta E}(\omega)= \\
& d\left[\sum_{i} z_{c_{i}} F \frac{G_{c_{i}}}{j \omega d+K_{c_{i}}}-\sum_{k} z_{a_{k}} F \frac{G_{a_{k}}}{j \omega d+K_{a_{k}}}\right]
\end{aligned}
$$

- la fonction de transfert, $\frac{\Delta m}{\Delta q}(\omega)$ :

$$
\begin{gathered}
=\frac{-\left(\sum_{i} m_{c_{i}} \frac{G_{c_{i}}}{j \omega d+K_{c_{i}}}+\sum_{k} m_{a_{k}} \frac{G_{a_{k}}}{j \omega d+K_{a_{k}}}+m_{s} \frac{G_{s}}{j \omega d+K_{s}}\right)}{j \omega\left[\sum_{i} z_{c_{i}} F \frac{G_{c_{i}}}{j \omega d+K_{c_{i}}}-\sum_{k} z_{a_{k}} F \frac{G_{a_{k}}}{j \omega d+K_{a_{k}}}\right]} \\
\frac{\Delta m}{\Delta q}(\omega)=j \omega \frac{\Delta E}{\Delta I_{F}} \frac{\Delta m}{\Delta E}(\omega) .
\end{gathered}
$$

De plus, il est possible de déduire deux fonctions de transfert partielles cationsolvant, $\frac{\Delta m_{c s}}{\Delta E}(\omega)$ et anion-solvant, $\frac{\Delta m_{a s}}{\Delta E}(\omega)$ en éliminant respectivement la contribution d'un anion et d'un cation à condition d'avoir la participation d'un cation, d'un anion et $\mathrm{du}$ solvant :

$$
\begin{aligned}
& \frac{\Delta m_{c s}}{\Delta E}(\omega)=\frac{\Delta m}{\Delta E}(\omega)-\frac{m_{a}}{j \omega} \frac{1}{F} \frac{\Delta I_{F}}{\Delta E}= \\
& -d\left(\left(m_{a}+m_{c}\right) \frac{G_{c}}{j \omega d+K_{c}}+m_{s} \frac{G_{s}}{j \omega d+K_{s}}\right)
\end{aligned}
$$

$$
\begin{aligned}
& \frac{\Delta m_{a s}}{\Delta E}(\omega)=\frac{\Delta m}{\Delta E}(\omega)+\frac{m_{c}}{j \omega} \frac{1}{F} \frac{\Delta I_{F}}{\Delta E}= \\
& -d\left(\left(m_{a}+m_{c}\right) \frac{G_{a}}{j \omega d+K_{a}}+m_{s} \frac{G_{s}}{j \omega d+K_{s}}\right) .
\end{aligned}
$$

Dans le cadre de notre travail, la contribution de trois espèces (anion, cation et solvant libre) qui interviennent lors de la compensation de charge, a été simulée dans les tableaux 1 et 2 en présentant les quatre fonctions de transfert générales $\frac{\Delta E}{\Delta I}(\omega), \frac{\Delta q}{\Delta E}(\omega)$, $\frac{\Delta m}{\Delta E}(\omega)$ et $\frac{\Delta m}{\Delta q}(\omega)$ et les deux fonctions partielles $\frac{\Delta m_{c s}}{\Delta E}(\omega), \frac{\Delta m_{a s}}{\Delta E}(\omega)$. La simulation d'une seule espèce participant au processus a été présentée dans d'autres publications [4,5]. En effet, pour compenser une charge positive créée par changement anodique du potentiel imposé au film, ce qui correspond au processus d'oxydation, trois possibilités sont offertes : soit un cation est expulsé, soit un anion est inséré ou soit les deux interviennent simultanément. Dans le premier exemple, dans les tableaux 1 a et $2 \mathrm{a}$, le solvant libre $\left(\mathrm{H}_{2} \mathrm{O}\right)$ est également échangé. Pour le diagramme d'impédance électrochimique $\frac{\Delta E}{\Delta I}(\omega)$, un demi-cercle est obtenu aux moyennes et hautes fréquences. Il correspond au transfert de charge en parallèle avec la capacité de la double couche. Une partie verticale aux basses fréquences, qui correspond au transfert ionique, est aussi déterminée. En calculant $\frac{\Delta q}{\Delta E}(\omega)$ d'après l'équation (9), les deux boucles obtenues nous montrent la participation des deux espèces chargées : à haute fréquence pour le cation et à basse fréquence pour $\mathrm{l}^{\prime}$ anion $\left(\mathrm{K}^{+}\right.$et $\mathrm{Cl}^{-}$ dans notre exemple). La fonction de transfert $\frac{\Delta m}{\Delta E}(\omega)$ fait apparaître trois boucles (une pour chaque espèce) : le premier demi-cercle (cation) est obtenu dans le troisième quadrant avec une valeur basse fréquence négative : dans le sens anodique $(\Delta E>0)$ le cation est expulsé $(\Delta m<0)$ ce qui donne un rapport $\frac{\Delta m}{\Delta E}(\omega \rightarrow 0)$ négatif. La deuxième boucle, à la suite du premier, en choisissant des constantes de temps bien séparées, correspond au solvant et enfin, la troisième dans le quatrième quadrant représente l'anion. 
Tableau 1. Représentation des quatre fonctions de transfert $\frac{\Delta E}{\Delta I}(\omega), \frac{\Delta q}{\Delta E}(\omega), \frac{\Delta m}{\Delta E}(\omega)$, et $\frac{\Delta m}{\Delta q}(\omega)$ dans le cas de la participation d'un anion et du solvant.

Table 1. Four transfer functions $\frac{\Delta E}{\Delta I}(\omega), \frac{\Delta q}{\Delta E}(\omega), \frac{\Delta m}{\Delta E}(\omega)$, and $\frac{\Delta m}{\Delta q}(\omega)$ when only one cation, one anion and one solvent participate to the electrochemical process.

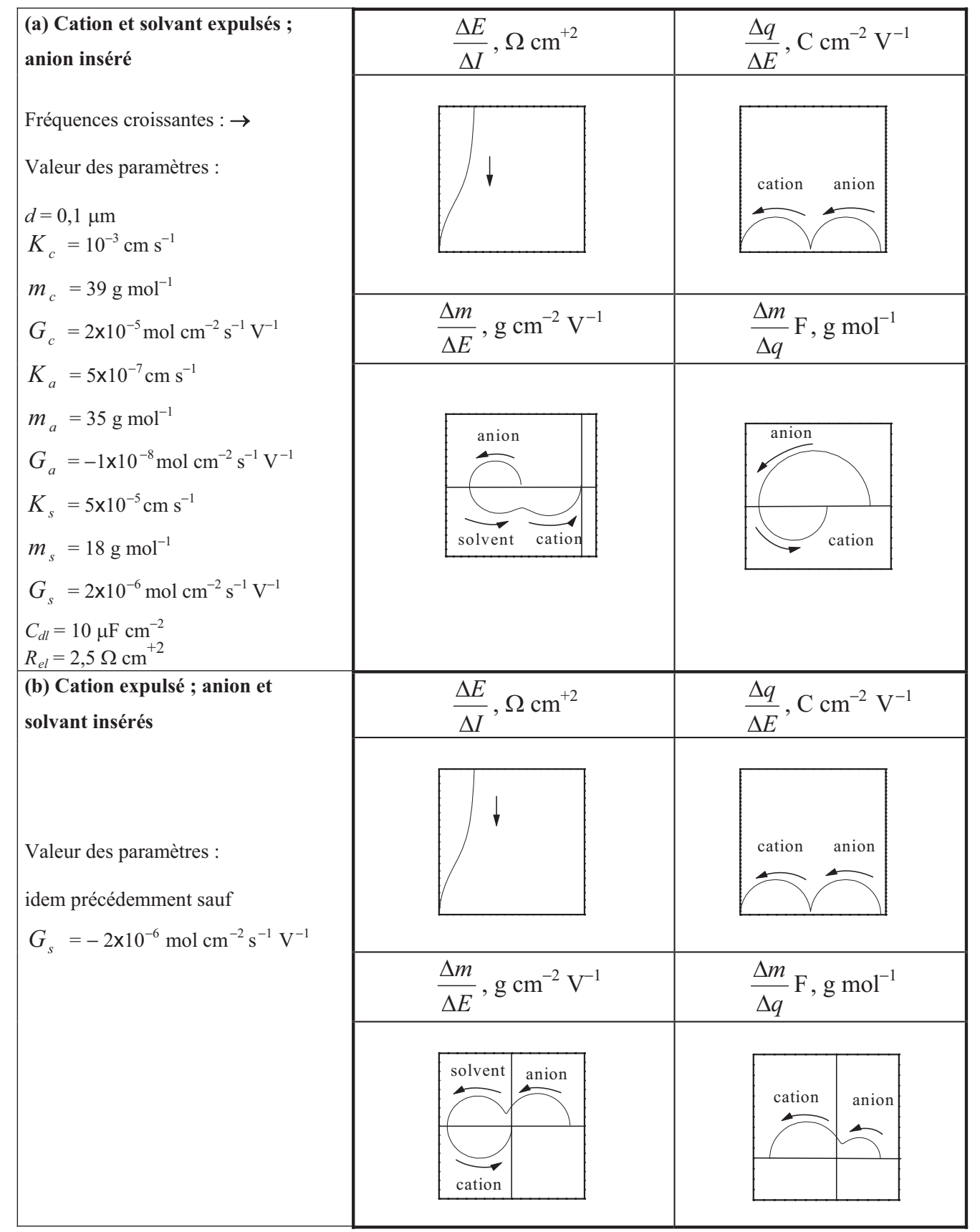

Deux boucles apparaissent pour $\frac{\Delta m}{\Delta q}(\omega)$ et correspondent à chaque ion. D'autre part, si le solvant est inséré lors de l'oxydation (Tab. 1b), $\frac{\Delta E}{\Delta I}(\omega)$ et $\frac{\Delta q}{\Delta E}(\omega)$ sont inchangées mais $\frac{\Delta m}{\Delta E}(\omega)$ fait apparaître la deuxième boucle relative au solvant dans le quatrième quadrant alors que $\frac{\Delta m}{\Delta q}(\omega)$ fait toujours apparaître deux boucles. Enfin, les deux fonctions partielles $\frac{\Delta m_{c s}}{\Delta E}(\omega)$ et $\frac{\Delta m_{a s}}{\Delta E}(\omega)$ (Tab. 2) sont calculées pour mettre en évidence le rôle de chaque espèce dans le processus réactionnel.

\section{Partie expérimentale}

\section{Microbalance à quartz en régime dy- namique}

De façon à utiliser une microbalance à quartz à $9 \mathrm{MHz}$ en mode alternatif, l'électrode de travail polarisée est soumise à une faible perturbation de potentiel. La réponse de la fréquence de la microbalance à quartz, $\Delta f$, correspondant à la modulation de masse, $\Delta m$, de l'électrode de travail, est mesurée 
Tableau 2. Représentation des deux fonctions de transfert partielles $\frac{\Delta m_{c s}}{\Delta E}(\omega), \frac{\Delta m_{a s}}{\Delta E}(\omega)$ dans le cas de la participation d'un anion et du solvant.

Table 2. Two partial transfer functions $\frac{\Delta m_{c s}}{\Delta E}(\omega)$ and $\frac{\Delta m_{a s}}{\Delta E}(\omega)$ when only one cation, one anion and one solvent participate to the electrochemical process.

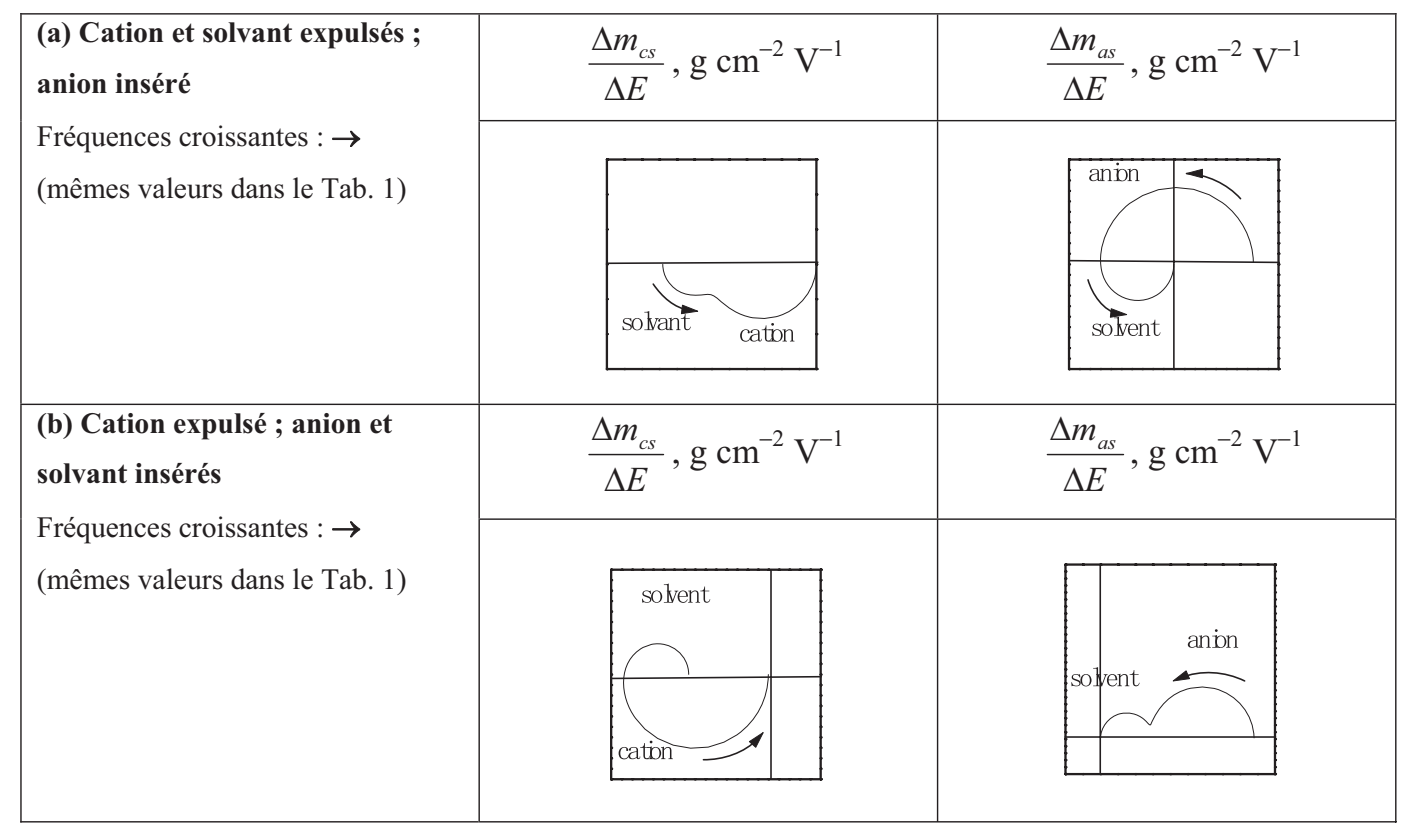

simultanément avec la réponse en courant de l'électrode $(\Delta I)$. Les signaux résultants sont envoyés à un analyseur de réponse en fréquence à quatre canaux (Solartron 1254), qui permet de mesurer simultanément la fonction de transfert électrogravimétrique, $\frac{\Delta m}{\Delta E}(\omega)$ et l'impédance électrochimique, $\frac{\Delta E}{\Delta I}(\omega)$.

\section{Préparation de bicouches bleu de Prusse/Nafion ${ }^{\circledR} 117$ sur l'électrode}

Tous les réactifs utilisés sont de qualité P.A. Les films de PB ont été obtenus par électrodéposition en mode galvanostatique à partir d'une solution $\mathrm{K}_{3}\left(\mathrm{Fe}(\mathrm{CN})_{6}\right)$ 0,02M, $\mathrm{FeCl}_{3}$ $0,02 \mathrm{M}$ et $\mathrm{HCl} 0,01 \mathrm{M}$. Un courant cathodique de $40 \mu \mathrm{A} / \mathrm{cm}^{2}$ a été appliqué pendant $150 \mathrm{~s}$. Une fois le film PB cyclé en milieu $\mathrm{KCl}$ $0,5 \mathrm{M}$ pH 2,8, $4 \mu \mathrm{L}$ du mélange de Nafion ${ }^{\circledR}$ 117 (Fluka-70160) et d'éthanol (ratio 2:5 en volume) est déposé au dessus du PB. En utilisant la technique de microscopie électronique à balayage, une estimation de l'épaisseur de la structure PB/Nafion ${ }^{\circledR} 117$ a été réalisée, celle-ci est d'environ $450 \mathrm{~nm}$. Puis, les mesures électrochimiques ont été effectuées différentes solutions : $\mathrm{HCl} 0,05 \mathrm{M}$ et $\mathrm{HCl}$ 0,05M+KCl 0,05M.

\section{Résultats et discussion}

Dans un premier temps, des mesures d'électrogravimétrie sur la structure $\mathrm{PB} / \mathrm{Nafion}{ }^{\circledR}$ 117 ont été réalisées en milieu $\mathrm{HCl}$ 0,05M $+\mathrm{KCl}$ 0,05M. Les différentes fonctions de transfert expérimentales sont présentées figure 2 ainsi qu'une simulation avec deux espèces et le solvant libre (Tab. 1) pour un potentiel de $0,1 \mathrm{~V} / \mathrm{ECS}$. L'allure de la courbe d'impédance reste identique à celle théorique donnée dans le tableau 1. La fonction de transfert $\frac{\Delta q}{\Delta E}(\omega)$, montre qu'il y a deux espèces chargées participant à la compensation de charge. Il est à noter que la contribution de l'anion apparaît comme une petite boucle à basse fréquence. Toutefois, cette contribution sera vérifiée par le calcul de la fonction de transfert partielle. En comparant les fonctions $\frac{\Delta m}{\Delta E}(\omega)$ et $\frac{\Delta m_{c s}}{\Delta E}(\omega)$, nous voyons que les deux boucles correspondant au cation et au solvant ne sont pas séparées, comme dans le tableau 1, car les deux constantes de temps sont certainement très proches l'une de l'autre. De plus, la quantité d'anion qui participe à la compensation de charge est beaucoup plus faible (Fig. 2f) que celles du cation et du solvant, sa contribution est difficilement discernée dans $\frac{\Delta m}{\Delta E}(\omega)$. Après avoir éliminé la participation du cation, la fonction partielle $\frac{\Delta m_{a s}}{\Delta E}(\omega)$ montre l'apparition de deux boucles : la première qui se situe dans le 

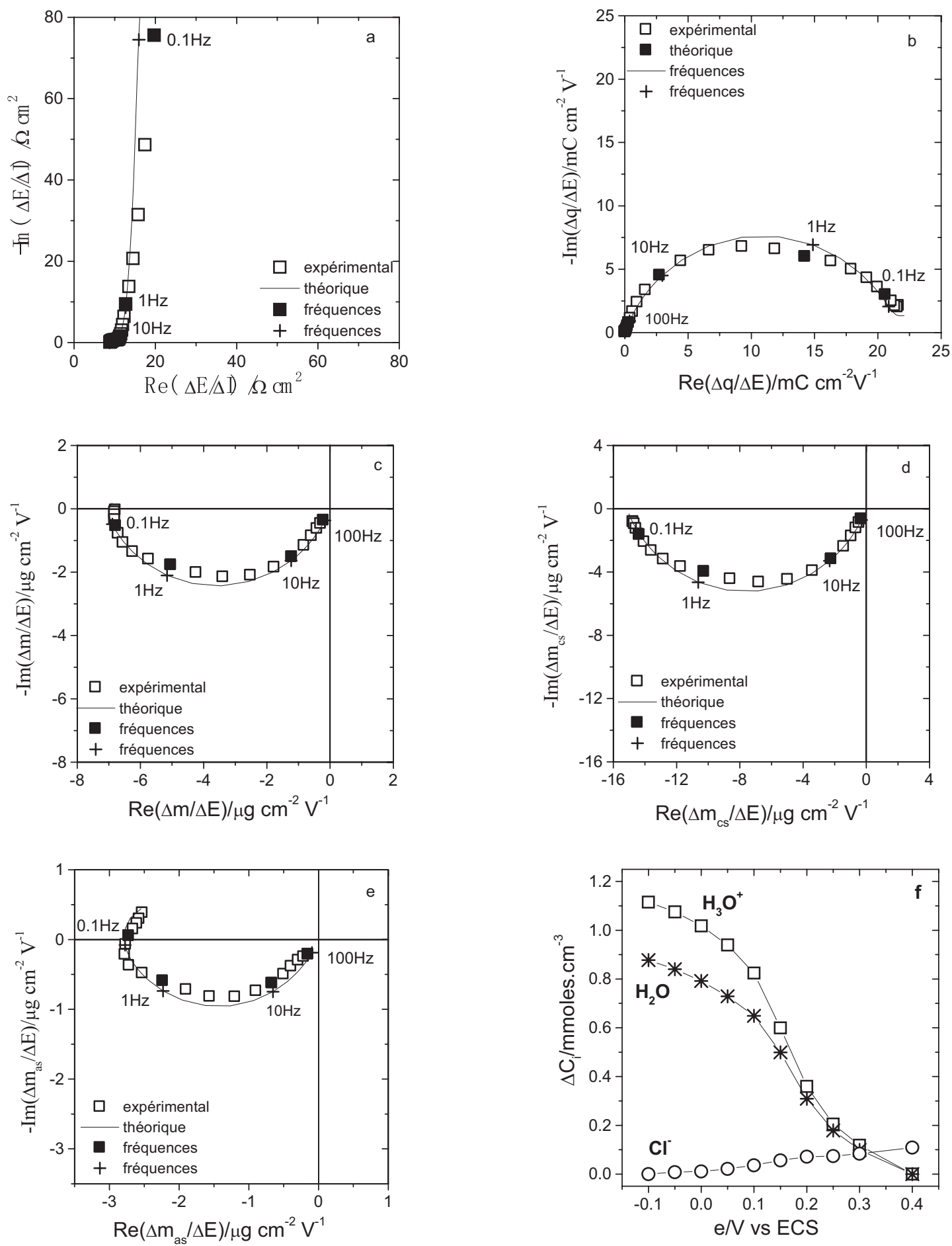

Fig. 2. $a, b, c$, d, e : Fonctions de transfert expérimentales et théoriques à 0,1 V/ECS et $f$ : variation de la concentration des espèces en tension pour $\mathrm{PB} / \mathrm{Nafion}{ }^{\circledR} 117$ dans $\mathrm{HCl} 0,05 \mathrm{M}$ et $\mathrm{KCl} 0,05 \mathrm{M}$.

Fig. 2. $a, b, c, d, e$ : Experimental and theoretical transfer functions at $0.1 \mathrm{~V} / \mathrm{ECS}$ and $f$ : species concentration changes over potential for $\mathrm{PB} / \mathrm{Nafion}^{\circledR} 117$ in $\mathrm{HCl} 0.05 \mathrm{M}$ and $\mathrm{KCl} 0.05 \mathrm{M}$.

troisième quadrant correspond au solvant et l'autre, dans le quatrième quadrant correspond à l'anion $\mathrm{Cl}^{-}$. Par conséquent, en accord avec la théorie (Tabs. 1 et 2), les trois espèces $\left(\mathrm{H}_{3} \mathrm{O}^{+}, \mathrm{Cl}^{-}, \mathrm{H}_{2} \mathrm{O}\right)$ participent au processus de compensation de charge : l'hydronium, le solvant sont expulsés alors que l'anion est inséré lors de l'oxydation de la structure $\mathrm{PB} / \mathrm{Nafion}{ }^{\circledR} 117$. D'autre part, la masse du cation calculée à partir de l'équation (10), correspond à l'hydronium $\mathrm{H}_{3} \mathrm{O}^{+}$ $(19 \mathrm{~g} / \mathrm{mole})$ pour tous les potentiels mesurés. Ceci est bien différent du cas du PB seul où le potassium $\mathrm{K}^{+}$intervient aux potentiels cathodiques et l'hydronium intervient aux potentiels anodiques. Ce phénomène peut être 

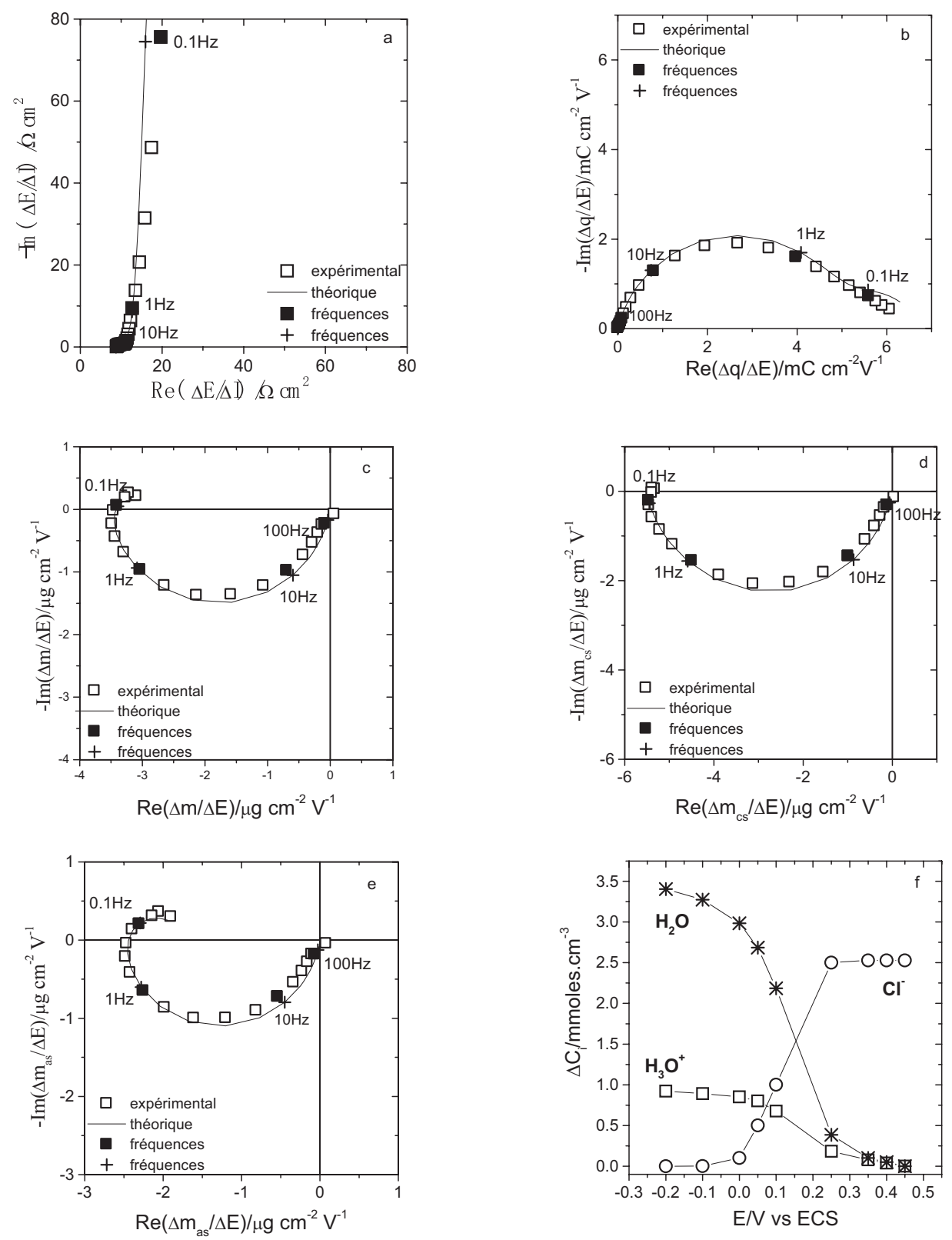

Fig. 3. $a, b, c$, d, e : Fonctions de transfert expérimentales et théoriques à $0,25 \mathrm{~V} / \mathrm{ECS}$ et $\mathrm{f}$ : variation de la concentration des espèces en tension pour $\mathrm{PB} / \mathrm{Nafion}{ }^{\circledR} 117$ dans $\mathrm{HCl} 0,05 \mathrm{M}$.

Fig. 3. $a, b, c, d, e$ : Experimental and theoretical transfer functions at $0.25 \mathrm{~V} / \mathrm{ECS}$ and $f$ : species concentration changes over potential for PB/Nafion ${ }^{\circledR} 117$ in $\mathrm{HCl}$ 0.05M.

expliqué par la propriété de perm-sélectivité du Nafion ${ }^{\circledR}$ 117, qui laisse l'hydronium passer plus facilement que le potassium. Quant à l'anion, qui est considéré comme ne pouvant pas être échangé avec le film de Nafion ${ }^{\circledR} 117$, son rôle peut s'expliquer par le maintien de l'électroneutralité dans le film, nous pouvons imaginer que son incorporation résulte de la trop faible expulsion du cation. Contrairement au cas du PB seul, la contribution du solvant n'est pas négligeable dans le cas de la structure $\mathrm{PB} / \mathrm{Nafion}{ }^{\circledR}$ 117. En effet, le Nafion ${ }^{\circledR} 117$ peut atteindre un certain niveau d'hydratation quand il est mis en contact avec un électrolyte. D'ailleurs, le nombre de molécules d'eau dans le Nafion ${ }^{\circledR} 117$ permet d'assurer le mouvement des protons au sein du film $\left(5 \mathrm{H}_{2} \mathrm{O}\right.$ par $\left.\mathrm{H}^{+}\right)$[1]. À partir des données de $K_{\alpha} G_{\alpha}$, les variations de concentration de chaque espèce, ont été calculées pour chaque potentiel (Fig. 2f). Quand la structure $\mathrm{PB} / \mathrm{Nafion}{ }^{\circledR} 117$ est étudié en milieu $\mathrm{HCl} 0,05 \mathrm{M}$, le rôle de chaque espèce se retrouve de la même façon que dans le milieu $\mathrm{KCl} 0,05 \mathrm{M}+\mathrm{HCl} 0,05 \mathrm{M}$. Cependant, la quantité relative de chlorure participant au processus de compensation de charge est 
plus importante comparativement au milieu précédent et aussi au cation pour le même milieu (Fig. 3f). Pour cette raison, la boucle de l'anion se présente de façon plus visible (quatrième quadrant) dans le diagramme de $\frac{\Delta m}{\Delta E}(\omega)$ (Fig. 3c). Dans les deux milieux, la contribution du solvant apparaît en premier, elle correspond à la constante de temps la plus élevée, 1 'hydronium apparaît en second et l'anion intervient dans le film avec la vitesse la plus lente.

\section{Conclusion}

L'étude par électrogravimétrie en régime dynamique de la structure PB/Nafion ${ }^{\circledR} 117$ dans deux milieux différents nous permet de connaître d'une manière aisée, pour chaque potentiel, le rôle des différents ions qui interviennent dans le processus de compensation de charge lors d'une réaction électrochimique. Afin de maintenir l'électroneutralité, la contribution de l'anion est à prendre en compte comme un des processus réactionnels lorsque la structure PB/Nafion ${ }^{\circledR}$ 117 est réduite ou oxydée. En contact avec un électrolyte, le Nafion ${ }^{\circledR} 117$, en principe conducteur protonique, permet néanmoins le déplacement d'anions à travers le film. De plus, le mouvement des cations (hydronium), qui assure majoritairement l'électroneutralité, est accompagné également du mouvement de solvant libre au sein du film de Nafion ${ }^{\circledR} 117$.

\section{Références}

[1] K.A. Mauritz, R.B. Moore, Chem. Rev. 104 (2004) 4535

[2] C. Gabrielli, J.J. Garcia-Jareno, M. Keddam, H. Perrot, F. Vicente, J. Phys. Chem. B 106, (2002) 3192

[3] C. Gabrielli, H. Perrot, A. Rubin, M.C. Pham, B. Piro, Electrochem. Comm. 9 (2007) 2196

[4] C. Gabrielli, J.J. Garcia-Jareno, M. Keddam, H. Perrot, F. Vicente, J. Phys. Chem. B 106 (2002) 3182

[5] J.J. Garcia-Jareno, A. Sanmatias, F. Vicente, C. Gabrielli, M. Keddam, H. Perrot, Electrochim. Acta 45 (2000) 3765

[6] J.J. Garcia-Jareno, D. Jiménez-Romero, F. Vicente, C. Gabrielli, M. Keddam, H. Perrot, J. Phys. Chem. B 107 (2003) 11321 